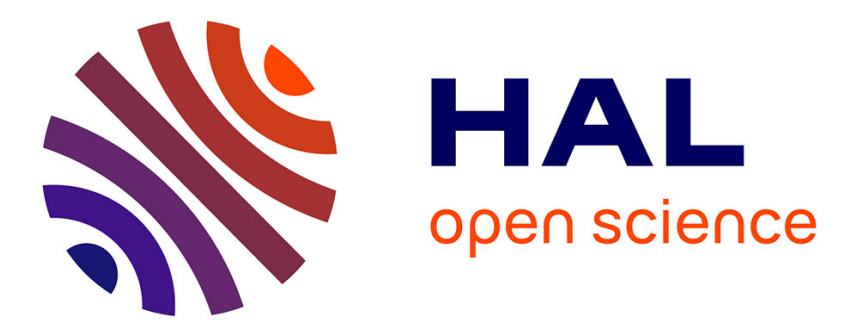

\title{
A Hybrid macro-micro pedestrians evacuation model to speed up simulation in road networks
}

Ngoc-Anh T. Nguyen, Jean-Daniel Zucker, Huu-Du Nguyen, Alexis Drogoul, Duc An Vo

\section{- To cite this version:}

Ngoc-Anh T. Nguyen, Jean-Daniel Zucker, Huu-Du Nguyen, Alexis Drogoul, Duc An Vo. A Hybrid macro-micro pedestrians evacuation model to speed up simulation in road networks. AAMAS, May 2011, Taipei, Taiwan. 10.1007/978-3-642-27216-5_28 . hal-00736474

\section{HAL Id: hal-00736474 https://hal.science/hal-00736474}

Submitted on 28 Sep 2012

HAL is a multi-disciplinary open access archive for the deposit and dissemination of scientific research documents, whether they are published or not. The documents may come from teaching and research institutions in France or abroad, or from public or private research centers.
L'archive ouverte pluridisciplinaire HAL, est destinée au dépôt et à la diffusion de documents scientifiques de niveau recherche, publiés ou non, émanant des établissements d'enseignement et de recherche français ou étrangers, des laboratoires publics ou privés. 


\title{
A Hybrid macro-micro pedestrians evacuation model to speed up simulation in road networks
}

\author{
NGUYEN Thi Ngoc Anh ${ }^{1,2,3}$, ZUCKER Jean Daniel ${ }^{1,2,3}$, \\ NGUYEN Huu Du ${ }^{4}$, Alexis Drogoul ${ }^{2,3}$, and Vo Duc An ${ }^{1,2,3}$ \\ 1 UPMC Univ Paris 06, UMI 209, UMMISCO, F-75005, Paris, France; \\ 2 IRD, UMI 209, UMMISCO, IRD France Nord,F-93143, Bondy, France; \\ 3 MSI, IFI, 42 Ta Quang Buu street,Hai Ba Trung District, Hanoi, Vietnam; \\ 4 University of Science, National University, Hanoi. \\ ngocanhfami@gmail.com, jean-daniel.zucker@ird.fr, \\ nhdu2001@yahoo.com, voducanvn@yahoo.com, alexis.drogoul@ird.fr
}

\begin{abstract}
The major methodologies of crowd simulation in a dynamic environments are either based on micro or macro models. These two types of models represent the trade-off between the level of detail and efficiency. The domain of pedestrian flow simulation on road networks is no exception and theories rely either on equation based model or agent based models. There is a growing interest in hybrid modeling that combines both of these types. This paper addresses the problem of combining both micro and macro models of pedestrians movement to speedup simulations. Hybrid model uses efficient macro modeling in part of the road networks that do not require a fine grained model and more detailed but less efficient micro modeling in critical locations. One key issue raised by such an approach and discussed is the consistency of the resulting hybrid model. Preliminary results presented in this article is a proof of concept that the use of hybrid model to simulate evacuation plan in road networks may be more efficient than the use of micro model alone.
\end{abstract}

Keywords: Crowd movement, Hybrid modeling, road networks, multi agent systems.

\section{Introduction}

Panic situations such as fire, bomb attack, tsunami, earthquake and among others in urban areas threaten more and more human livings and challenge politicians to decide appropriate preparation. Taking the right actions at the right time will make evacuation plan better for everyone involved. Therefore, it is need to design good instructions for people to follow in such panic situations. The challenge is how can the authorities create the proper instructions? One possible way is to simulate panic situations in libraries. In fact, the application of simulations is becoming essential to devise evacuation plans in several projects [11],[2]. Currently, the simulation of panic situation plays an important role in order to design a good decision support for the authorities. The population and 
the city are prepared to guarantee that there will be the least possible avoidable causalities.

Evacuation simulation in case of a Tsunami for example can be used to explore different questions: a) what is the efficiency of each pedestrian behaviors ? b) what is the global number of survivors given a particular set of instructions and road signs ? c) what is the impact of having stubborn individuals that do not follow signs ? etc. The response to these questions may then be used to support decisions made by the authorities to better prevent or handle evacuation process. Normally, there is a crowed population with many dangerous resources in urban areas. Therefore, if hazard situations take place in urban areas, there is a lot of vulnerability. Since urban areas include complicated road networks, evacuation in these road networks is a difficult problem that need to be researched. In addition, each urban areas has its own peculiarities due to its topology, its infrastructure, its road network, etc. Real road networks of many cities are now available and represented in Geographic Information Systems (GIS) systems supporting realistic simulations.

One motivation of this work is to develop simulation tools to prepare Vietnamese city such as the city of Nhatrang to become Tsunami ready like many Americans or Japanese city are now already. Nhatrang city, a tourism beach, locates in the middle of Vietnam. This city is vulnerable to tsunamis as it is closed to the earthquake epicenters of the Philippine islands. Optimizing the evacuation plans in Nhatrang city is thus a term issue. More formally, we investigate the problem of simulating evacuation on a real GIS road networks with $J$ junctions, $B$ different safe places that pedestrians ought to reach to escape.

On one hand, a macro type is used model the situation. Macro models of evacuation problems are often described using fluid dynamic equations. The environment of these models is often considered as homogeneous and the fluid system dynamics is represented by the density of evacuees. The road network in a macro environment is considered a finite directed graph. The solution of a macro model usually requires many assumptions that do not match at all human behaviors. The advantage of such model lie in their tractability. On the other hand, we use micro type, i.e. agent-based models. Normally, these models consider details behaviors of agents. In this case, each pedestrian can be considered as an agent. Each agent has its own behaviors such as its own action (perception, move,), interaction with others agents and interaction with environment. Contrary to macro models, environment in a micro model is heterogeneous. Here, heterogeneous environment GIS is described in the agent based model. The weak points of such models are data and huge computation time. In addition, the drawback of such models is related to the tractability of the simulation when scaling up to realistic population.

In this contribution, we present an approach using Hybrid Modeling in order to combine the efficiency of Macro Modeling (equation-based modeling) and the advantages of Micro Modeling (agent based modeling) in the study of the evacuation problem. The paper is organized as follows. Section 2 presents the two models for the evacuation problem. It is structured into two subsections. 
Subsection 2.1 presents a macro model, subsection 2.2 presents a micro model. Section 3 presents a hybrid evacuation flow model which combines the micro and macro models in section 2. Section 4 presents an implementing of the models. Section 5 presents a conclusion and some perspectives for future works.

\section{The approaches applied in pedestrian flow}

In large scale environment, all areas are not of equal importance w.r.t to the problem considered. Humans for example in such panic situation have more difference in behaviors at junction (where a decision must be taken) than along a road when trapped in a traffic jam. We suggest to use a micro model in such important location of the map where the individual behavior is particularly relevant to the modeling. On the contrary when the individual behavior is less critical we suggest using a macro model. The hybrid model is the set of these two models.

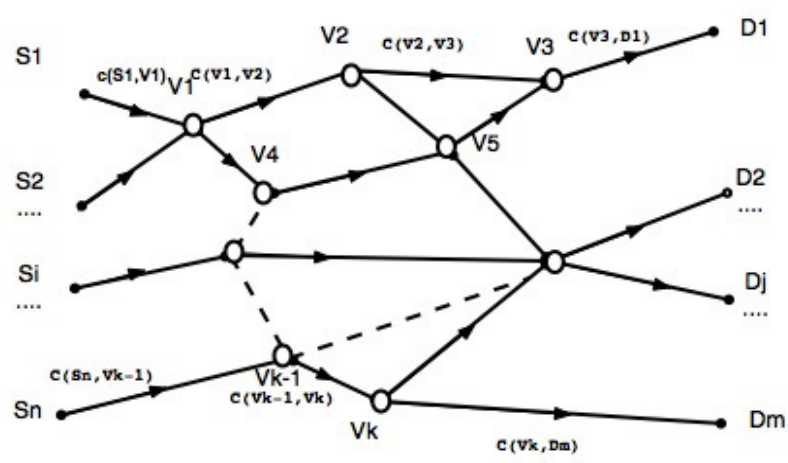

Fig. 1. road networks are modeled as directed graph.

The road network in macro model is represented as a finite directed graph $\mathcal{G}=(\mathbf{E}, \mathbf{V})$. Edges and vertices of the directed graph are roads and junctions of the road network respectively. $\mathbf{E}$ is the set of roads and $\mathbf{V}$ is the set of junctions. At the junction $V_{\alpha} \in \mathbf{V}$, let $\delta_{\alpha}^{-}\left(r e s p . \delta_{\alpha}^{+}\right)$the set of indices of all the incoming roads to $V \alpha$ (resp. outgoing roads from $V \alpha$ ). Every edge $(u, v) \in \mathbf{E}$ has a nonnegative, real-valued capacity $c(u, v)$. If $(u, v) \notin \mathbf{E}$, we assume that $c(u, v)=0$.

The same road networks in our micro model is represented by a GIS with metadata about roads, junctions, buildings, rivers, bridges, obstacles, etc.

\subsection{Macro model}

This subsection deals with a macro model of pedestrian flow on a road networks. The pedestrians are homogeneous with with a space- time continuum. More pre- 
cisely, the conservation law formulation proposed by Lighthill, Whitham and Richards (LWR) [9] is represent the fluid dynamics by partial difference equations. This nonlinear framework is based simply on the conservation of density pedestrians in one road and is described by the equation:

$$
\frac{\partial p}{\partial t}+\frac{\partial}{\partial x} f(p)=0
$$

where

$$
f(p)=p v(p), v(p)=v_{\max }\left(1-\frac{p}{p_{\max }}\right) .
$$

and $(t, x) \in \mathbf{R} \times \mathbf{R}_{+}$are time variable and spatial variable. $p=p(x, t) \in$ $\left[0, p_{\max }\right]$ is density of pedestrians, $v=v(t, x)$ is the average velocity pedestrians and $f(p)=p v(p)$ is the pedestrian flow. if there is an initial value, the equation (1) is called Riemann. The initial values is chosen as following

$$
p(x, 0)= \begin{cases}p_{l} & \text { if } x \leq 0 \\ p_{r} & \text { if } x>0\end{cases}
$$

where $p_{l}, p_{r}$ are two parameters being constant values.

Because the function $f(p)$ is concave, the weak solution of the Riemann problem that published in [6], [9] is.

(i) if $p_{l}<p_{r}$ the solution including a shock wave is given by

$$
p(x, t)= \begin{cases}p_{l} & \text { if } x \leq v_{\max }\left(1-\frac{p_{r}+p_{l}}{p_{\max }}\right) t \\ p_{r} & \text { if } x>v_{\max }\left(1-\frac{p_{r}+p_{l}}{p_{\max }}\right) t .\end{cases}
$$

(ii) if $p_{l}<p_{r}$ the solution of the equation is

$$
p(x, t)= \begin{cases}p_{l} & \text { if } x \leq v_{\max }\left(1-\frac{2 p_{l}}{p_{\max }}\right) t \\ \frac{p_{\max } v_{\max }}{2 v_{\max }+p_{\max }} & \text { if } v_{\max }\left(1-\frac{2 p_{l}}{p_{\max }}\right) t \leq x \leq v_{\max }\left(1-\frac{2 p_{r}}{p_{\max }}\right) t, \\ p_{r} & \text { if } x>v_{\max }\left(1-\frac{2 p_{r}}{p_{\max }}\right) t\end{cases}
$$

(iii) if $p_{l}=p_{r}$ the solution is constant and given by: $p(x, t)=p_{l}$.

The pedestrian flow in road networks is investigated in [9]. Each road $i$ is modeled by an interval $I_{i}=\left[a_{i}, b_{i}\right]$, possibly with either $a_{i}=-\infty, b_{i}=+\infty$. In the case of the LWR model the conserved quantity is the variable $p_{i}=p_{i}(x, t)$ : $I_{i} \times \mathbf{R}_{+} \rightarrow \mathbf{R}$, so that on each edge $i$ of the graph, the pedestrian flow is governed by the following scalar conservation law:

$$
\frac{\partial p_{i}}{\partial t}+\frac{\partial}{\partial x} f\left(p_{i}\right)=0, \forall i \in \mathbf{I}
$$

where 


$$
f\left(p_{i}\right)=p v\left(p_{i}\right), v\left(p_{i}\right)=v_{i, \max }\left(1-\frac{p_{i}}{p_{i, \max }}\right), \forall i \in V .
$$

In addition, the initial values of road $i$ are two constant values, i.e

$$
p_{i}(x, 0)= \begin{cases}p_{i, l} & \text { if } x \leq 0, \\ p_{i, r} & \text { if } x>0 .\end{cases}
$$

The solution in each road has the same formulas with the case of one road represented above. This model is appropriate to reveal shock formation as it is natural for conservation laws whose solutions may develop discontinuities in finite time even for smooth initial data. However, the behaviors of pedestrians are different but they can not be represented in the LWR model. The importance of behaviors in evacuation are investigated by agent based model. In addition, at junctions of road networks the dynamics of pedestrian flow is complex so micro model will be used and be investigated in the next subsection.

\subsection{Micro model}

This subsection deals with a micro model of pedestrian flow on a road networks. To present this micro model, we rely on the Overview Design concepts Detail (ODD) protocol $[3,4]$ which is widely accepted as an agent based model template.

\section{Overview}

\section{- Purpose}

The purpose of this overview is to detail each pedestrian's behavior facing a Tsunami alert sand then to link the behavior to both the probability to survive and the global amount of time spend to evacuate. The simulation of the behaviors produces the emergence of pedestrians moving along the road networks. For the sake of simplicity we have only considered two kinds of behaviors, one that corresponds to an knowledgeable pedestrian (called a Fox) and another one that is more a followers that do not have any idea how to respond to a Tsunami alert.

- Entities, state variables and scales

First, the pedestrians are entities. A fox agent is defined as a pedestrian who has been trained on evacuation procedures or has knowledge of how get to the safe place efficiently. The pedestrian evacuating randomly or following one fox agent is called sheep agent.

The state variables of pedestrian are their position and velocity. Each agent has his own behavior according to his goal and knowledge of the environment. His perception in the environment depends on space that the agent can observe. Position of the other agents around, road infrastructure can all be used by the agent to decide its move. His decisions are based on his perception of the environment and the information shared with the other agents. As agent moves, he 
adapts his speed to reach his desired speed. Indeed, if his speed is less than the desired speed, and if there is enough space, he will try to accelerate.

Second, spatial scale in this model is meter and the unit time is minute. We consider the time that all the pedestrians move to the safe places and the specific area.

- Process overview and scheduling

If he is a sheep agent, he choose a road randomly at a junction. If he is a fox agent, he moves to the direction that correspond to the shortest path to its objective position.

In panic situations, the pedestrians always want to escape from the dangerous place as quickly as possible. If there are no information available, they often move randomly or follow the crowd. The road networks is complex so the sheep agent finds it difficult to finding the safe place or reaching the safe place before its too late. Diagram (Fig. 2) is described the process of agents in evacuation.
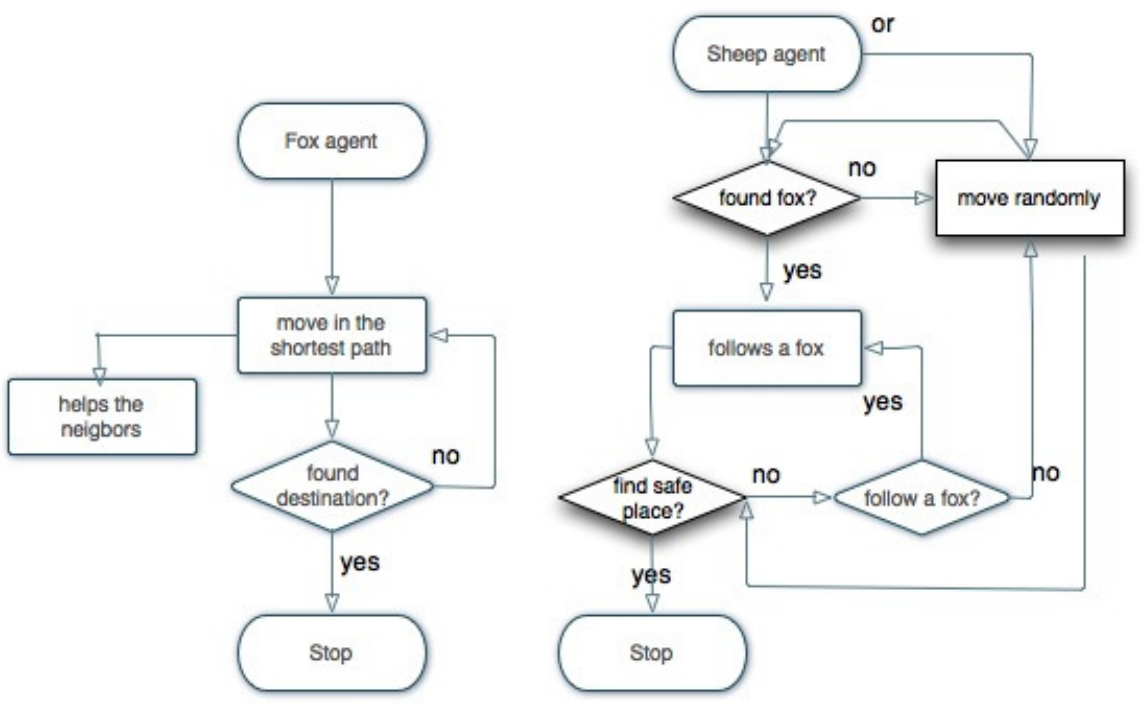

Fig. 2. Process of sheep agent and fox agent in moving

\section{Design concepts}

- Basic principles

The agents follow one direction to safe place. The fox agent chooses the shortest path and sheep agent moves randomly or follows one of the fox agents. 


\section{- Emergence}

The result shows that the more the number of fox agents in population, the lesser time spending of all agents.

- Adaptation

We consider an agent $\gamma$ arbitrary. His velocity depends on the neighbors ahead of him and the capacity of the road. If the number of neighbors are greater than the critical value then he can not move forward so the velocity is equal 0 . If the neighbors are crowd then he moves slowly, on the contrary if the neighbors are few then he moves fast on his own. His velocity is represented:

$$
v(\gamma)=v_{\text {average }}\left(1-\frac{\text { neighbors }(\gamma)}{\text { critical }}\right)
$$

where critical depends on the capacity of the road and the local density of the agent $\gamma$. Each agent will have an argument about the position, velocity, different goals, the circle observation, his decision choose direction when he stands at the junctions.

\section{- Objectives}

The fox agent's objective is to find the shortest path from current position to one of safe places and help the sheep agents. The objective of sheep agent is to find a fox agent and explore a safe place when he can not find any fox agent.

\section{- Prediction}

The fox agent can predict the block of the traffic when he senses the crowd. The prediction of the fox agent helps him and the followers evacuate more effectively.

\section{- Sensing}

There are two levels in sensing the environment. The high level sensing of an agent is to know the sequence of roads from his position to one of destinations. The low level sensing of an agent is the local environment that helps him avoid the obstacles, the crowd and move efficiently on the road. A fox agent has both the high and the low levels. The high level sensing shows an fox agent the shortest path. On another hand, a sheep agent has only low level sensing or he tries to find an fox agent.

\section{- Interaction}

The sheep agent tries to find a fox agent near him and he follows. All agents impact each other by adapting their velocity.

\section{- Collectives}

The group includes a fox agent and all the sheep agents following the fox agent. The fox agent of each group is the leader that helps all the members of the group to escape danger to the safe place. 


\section{Detail}

- Initialization

The initial data are the number of agents evacuating, the number of safe places, the road networks is represented GIS environment.

- Input data

Input data are the densities and velocities of sources. The proportion of fox agent in the population.

\section{Hybrid evacuation flow model on road networks}

\subsection{Environment of the hybrid models}

The large scale environment of a city is represented by GIS needs much computation cost. There are some areas that are very important in evacuation whereas other areas can be ignored. The unimportant areas are represented by a direct graph in macro model and the important areas are represented by the GIS in micro model. In the environment of the hybrid model, each road is separated by three patches. On patches 1 and 3 of each road, a micro model is considered, whereas on patch 2 a macro model is of the pedestrian flux is considered. The hybrid environment described in (Fig. 3) combines a direct graph and GIS.
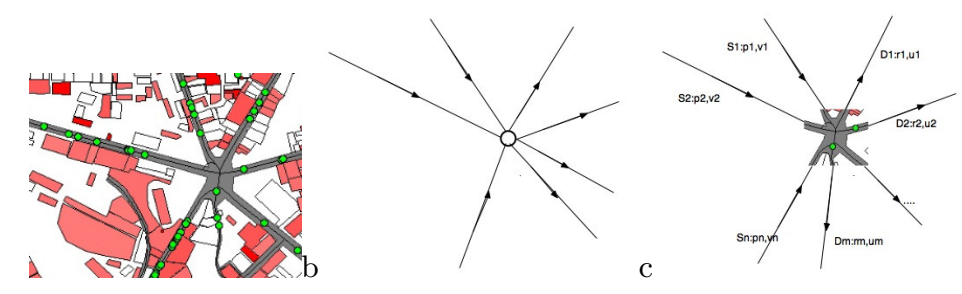

Fig. 3. a, The GIS environment in the micro model; b, the abstract environment representing direct graph in macro model; c, hybrid environment in hybrid model.

\section{- Micro patch 1}

Pedestrians are simulated by agent based model that each agent is behaving as a fox or a sheep (depending on his type). The simulation in this patch is represented within a micro model on top of a map represented in a GIS.

- Macro patch 2 
This patch is a stretch of the road. In the hybrid model, this patch is described by an edge of a direct graph with two parameters that are the length of this patch $L_{i}$ and capacity $c_{i}$ of the road $i$. The patch 2 has two special positions: its extremities. the first one correspond to the change from patch 1 to patch 2 . It is called the source and the position changing from patch 2 to patch 3 called destination.

- Micro patch 3

This patch is represented the same as patch 1 .

\subsection{Interfacing the two models}

As mentioned above, one of the key issue in combining two such models is the coherence of the results in terms of time, space (discrete/continuous) and behaviors. Let us divide each road into three patches: the first extremity, the main central part and the second extremity. A junction corresponds to a set of patch 3 from the entering roads to a set of patch 1 of the exiting roads. Two questions arise, the transfer from a micro agent into the continuous one (from patch 1 to patch 2 ) and the transfer from the continuous patch (2 to patch 3 ). The first transition may be seen as a dematerialization of the agent, the second as a rematerailization.

Transition from micro to macro models The first transition we consider is that of a micro pedestrians transferring towards the macro patch. Let us choose one arbitrary road $i$ and let us consider the problem in detail :

- The interval of the time from $t$ step to $t+1$ step is called the time step $t$ th

- The aggregation triggers changes the number of pedestrians of the micro model to the flow of pedestrian as the parameters $p_{i}(t$, source) of macro that is investigated in patch 2 .

- The average velocity of the number pedestrians at source gives the velocity of flow pedestrian in the macro patch $2 v_{i}(t$, source $)$.

Transition from macro to micro models (Fig. 4) illustrates the transition from the macro patch. The macro patch referred to as the tube. The length of the macro patch of road $i$ is denoted $L_{i}$.

- The macro values $p_{i}(t$, source $), v_{i}(t$, source $)$ of source is used for the the initial of macro model. The average time flow of pedestrian to pass the tube of the road $i$ is

$$
T(t)=\frac{L_{i}}{v_{i}(t, \text { source })} .
$$

Applying the solution of the formula for the macro model (4), (5) and (6), at the time $t+T(t)$ gives results on the density and velocity of pedestrians flow at the destination $p_{i}(t+T(t)$, destination $), v_{i}(t+T(t)$, destination $)$. 


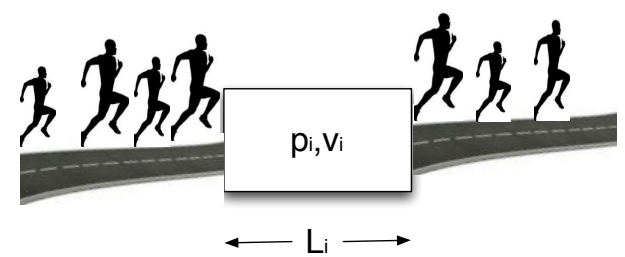

Fig. 4. The macro patch of the road $i$ is described by a tube. The length of the tube is denoted $L_{i}$. The begin and the end of the road $i$ are simulated by the micro model and the tube is simulated by the macro model.

The flow of pedestrian at $t+T(t)$ is

$q_{i}(t+T(t)$, destination $)=p_{i}(t+T(t)$, destination $) \cdot v_{i}(t+T(t)$, destination $)$.

These results are the parameters of micro model for the patch 3. First, we assume the flow of pedestrians is a Poisson process used in [10], [8]. Using the Poisson distribution with parameter $\lambda=$ mean $=q_{i}(t+T(t)$, destination $)$ at the destination generates the number of pedestrian agents.

- The velocity of each pedestrian is generated by the normal truncated distribution with the $\mu=$ mean $=v_{i}(t+T(t)$, destination $)$, at destination.

- The time for one agent order $k$ th comes into the tube at the time $t$ is released out of the tube to the patch 3 :

$$
t+T(t)+g\left[k, q_{i}(t+T(t), \text { destination })\right] .
$$

where $g\left[k, q_{i}(t+T(t)\right.$, destination $\left.)\right]$ is value of Gamma distribution

This formula is based the theory of Poisson process that we can read in [13][14]. The arrival time of agent order $k$ follows Gamma distribution with parameter $q_{i}(t+T(t)$, destination $)$.

\section{Implementing the model}

\subsection{Simulation of the micro model}

The data in Nhatrang are used to simulate the evacuation of the pedestrians. The data in Nhatrang are metadata including the road networks, buildings, beach, rivers, sea and safe places calling targets. Parameters of the model are the numbers of foxes, sheep, average and variance velocity of pedestrians. The simulation of the model is showed in (Fig. 5)

The simulation gives us the average time it takes an agent from his initial position to one of the safe targets. In addition, the important result is the number of the agents who reach the targets. These results are shown in (Fig. 6).

The results of simulation help us analyzing the relationship between the number of fox, sheep agents with the number of agents reaching the targets and 

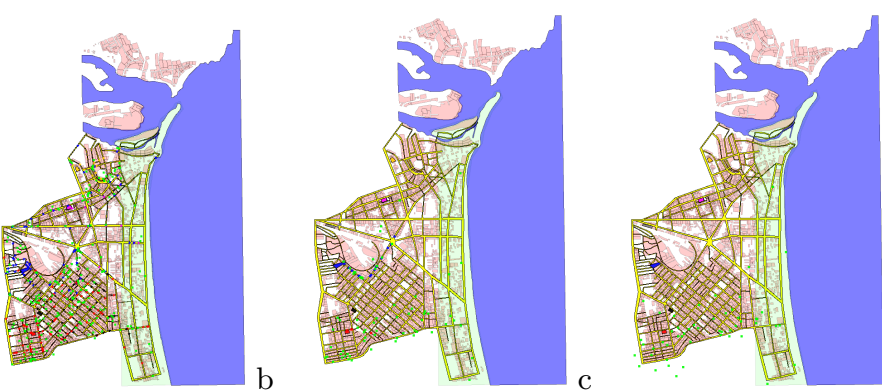

Fig. 5. The shape of the fox agent is circle, the shape of sheep agent is square. The fox agent chooses one of the safe places to reach. Each safe place has a different color from the other safe places. A fox agent has a unique corresponding to the color of his safe place. The above results use constant mean velocity $10 \mathrm{~m} / 7.5$ seconds and variance velocity $1 \mathrm{~m} / 7.5$ seconds (the unit of the time in simulation is 7.5 seconds). These three figures describe the simulation with respect to the time step: a. $T=1$, b. $T=150$, c. $T=350$.
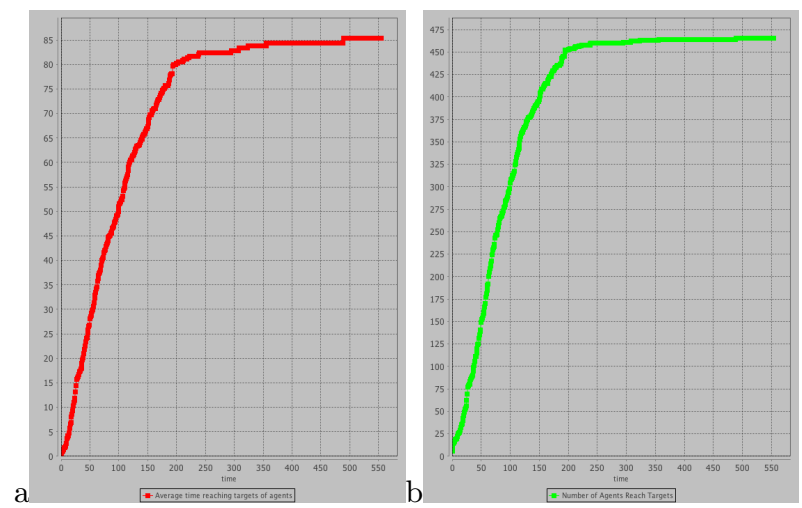

Fig. 6. a. The figure shows the average time that one agent spends from his initial position to the target. At the time step 555 , the average time converges 85 steps. b. The figure represents the number of agents that reach the targets. At the time 555, the number of agent reach the target is converges 460 agents 
the average time needed. (Fig. 7 a) describes the simulation result with different numbers of fox and sheep agents. (Fig. $7 \mathrm{~b}$ ) shows the function of the number of agents that reach the safe targets with the numbers of fox and sheep agents variables using a linear regression.
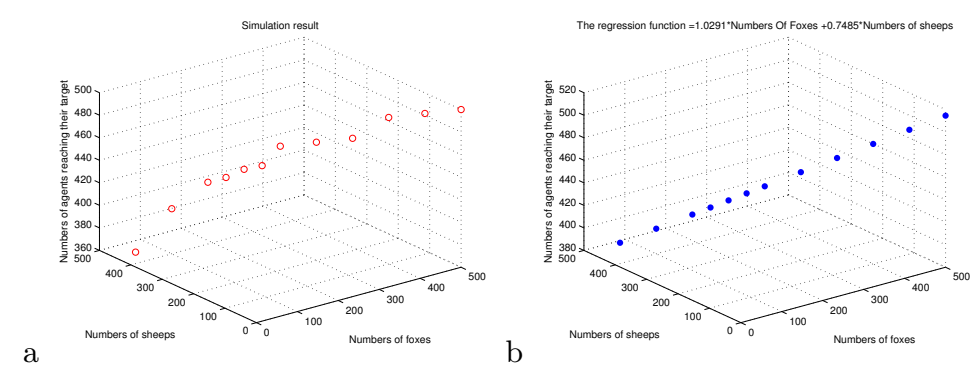

Fig. 7. The above result uses a constant mean velocity $10 \mathrm{~m} / 7.5$ seconds and variance velocity $1 \mathrm{~m} / 7.5$ seconds. a. a shows the simulation result of the number of agents reaching the targets. b. shows an analysis result of the agents reaching the target by using the linear regression.

\subsection{Simulation of the hybrid model}

To simplify the program, we consider a road networks having 9 roads as shown in (Fig. 8)

The environment of hybrid model is represented by a combination of the GIS at the end and the begin of each road and the direct graph along the stretch of each road in (Fig. 8). In the fact that, the micro model is simulated at the begin and the end of the road and stretch is simulated macro model. Therefore, all junctions are investigated in detail in the micro model.

\section{Conclusion}

The problem of speeding up very large environment in agent based model such as the ones used in crowd simulation is key to support realistic Decision Support Systems. In this paper we have shown an approach to Hybrid modeling for evacuation simulation. The key idea is to exploit the advantages from both macro and micro modeling. The two methods are defined to exchange agents at the source and the destination of a road. A case study to prepare the City of Nhatrang to become Tsunami ready based on hybrid modeling shows that it not only offers more efficient execution than micro modeling, but also improves the simulation quality in comparison with macro modeling. The results presented are yet to be extended to very large simulations including hundred of thousands of pedestrians. Future work includes large scale simulation and exploring various emergent behavior resulting from various types of behaviors. 


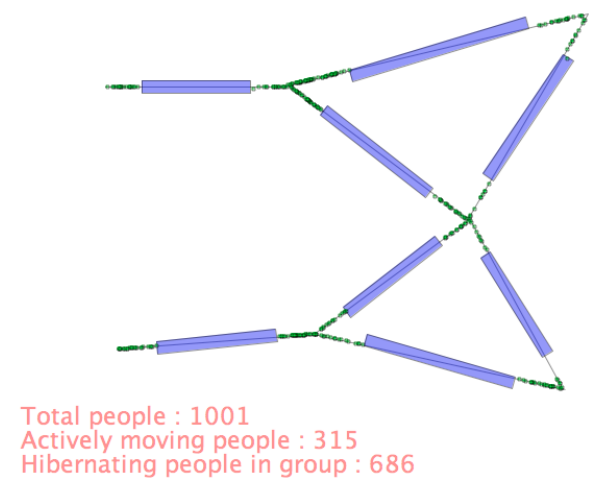

Fig. 8. Micro model of the road networks and Hybrid model of road networks are implementation. The number of people hibernating (not simulated in the ABM) are proportional to the speedup provided by the hybrid modeling. Indeed, only the fraction of the total pedestrians are effectively consuming simulation CPU.

\section{References}

1. Flotterod, G., Nagel, K., "High Speed Combined Micro/Macro Simulation of Traffic Flow" Intelligent Transportation Systems Conference, 2007. ITSC 200\%. IEEE pp. 1114 - 1119, Seattle, WA, Sept. 30 2007-Oct. 32007.

2. Gianluca Antonini, Michel Bierlaire, Mats Weber ,"Discrete choice models of pedestrian walking behavior " Transportation Research Part B, Volume 40, pp 667 687,2006

3. Volker Grimm and Uta Berger and Finn Bastiansen and Sigrunn Eliassen and Vincent Ginot and Jarl Giske and John Goss-Custard and Tamara Grand and Simone K. Heinz and Geir Huse and Andreas Huth and Jane U. Jepsen and Christian Jorgensen and Wolf M. Mooij and Birgit Muller and Guy Peer and Cyril Piou and Steven F. Railsback and Andrew M. Robbins and Martha M. Robbins and Eva Rossmanith and Nadja Ruger and Espen Strand and Sami Souissi and Richard A. Stillman and Rune Vabo and Ute Visser and Donald L. DeAngelis, "A standard protocol for describing individual-based and agent-based models" Ecological Modelling,volume 198, number 1-2, pp115 - 126, 2006.

4. Volker Grimm and Steven F. Railsback, "Individual-based Modeling and Ecology" Princeton University Press, Princeton, New York, 2005.

5. Gunnar G.Lovas "Modeling and simulation of pedestrian traffic flow", Transportation Research Part B, Volume 28, No 6, pp 429 -443, 1994.

6. Jennifer McCreaa, and Salissou Moutari, "A hybrid macroscopic-based model for traffic flow in road networkss" European Journal of Operational Research Volume 207, Issue 2,pp. 676-684, 1 December 2010.

7. L. Leclercq, S. Moutari, "Hybridization of a class of second order models of traffic flow" Simulation Modelling Practice and Theory, Volume 15, pp 918 - 934, L. Leclercq a, 2007. 
8. Laurent Magne, Sylvestre Rabut, Jean Francois Gabard, "Towards an hybrid macro-micro traffic flow simulation model" NFORMS Salt Lake City String 2000 Conference May 7-10, 2000.

9. Ludovic Leclercq, "Hybrid approaches to the solutions of the Lighthill Whitham Richards model" Original Research Article Transportation Research Part B: Methodological, Volume 41, Issue 7, Pages 701-709, August 2007.

10. Mohamed said El Hmam, Daniel Jolly, Hassane Abouaissa, Amar Benasser, "Modelisation hybride du flux de trafic", Methodologies ET Heuristiques pour l'Optimisation des Systemes Industriels, Mohsi, pp 193-198, janvier, 2008.

11. Markos Papageorgiou, Jean-Marc Blosseville, Habib Hadj-Salem, "Marcroscopic modelling of traffic flow on the boulevard Peripherique in Paris", Transportation Research Part B, Volume 23B, No1, pp $29-47,1989$.

12. Ellen-Wien Augustijn-Beckers, Johannes Flacke, Bas Retsios, "First International Conference on Evacuation Modeling and Management Investigating the effect of different pre-evacuation behavior and exit choice strategies using agent-based modeling", Procedia Engineering, Volume 3, pp 23 35, 2010.

13. Sheldon M. Ross, "Introduction to Probability Models", A Harcourt Science and Technology Company, Sixth Edition, 2006.

14. William Feller, "An Introduction to Probability Theory and Its Applications", Wiley series in probability and mathematical statistic Volume II, 1971. 